\title{
New Formulas for Irregular Sampling of Two-Bands Signals
}

\section{Bernard Lacaze}

Telecommunications for Space and Aeronautics, Toulouse, France.

Email: bernard.lacaze@tesa.prd.fr

Received March 15 $5^{\text {th }}, 2011$; revised June 20 $0^{\text {th }}, 2011$; accepted June $30^{\text {th }}, 2011$.

\begin{abstract}
Many sampling formulas are available for processes in baseband $(-a, a)$ at the Nyquist rate $a / \pi$. However signals of telecommunications have power spectra which occupate two bands or more. We know that PNS (periodic non-uniform sampling) allow an errorless reconstruction at rate smaller than the Nyquist one. For instance PNS2 can be used in the two-bands case $(-a,-b) \cup(b, a)$ at the Landau rate $(a-b) / \pi$ We prove a set of formulas which are available in cases more general than the PNS2. They take into account two sampling sequences which can be periodic or not and with same mean rate or not.
\end{abstract}

Keywords: Stationary Processes, Irregular Sampling, Two-Bands Processes

\section{Introduction}

Communications signals are often transmitted in frequency channels which are in the form $(b, a)$ where the width $(a-b)$ is small with respect to $a$. For instance we have $(a-b) / a \sim 0.2$ for the whole FM band $(87.5$ $\mathrm{MHz}, 108 \mathrm{MHz}$ ). Of course, the occupied frequency interval by a single station is smaller which leads to relative occupency very weak. When modelling by stationary processes, negative frequencies are taken into account. Real processes are transmitted in frequency bands in the form $(-a,-b) \cup(b, a)$. We know that such processes can be reconstructed by sampling at the Landau rate $2(a-b) / \pi$, which is weaker than the Nyquist rate $2 a / \pi$ [1]. PNS2 (Periodic Nonuniform Sampling of order 2) is the most known example [2,3]. In the case of baseband signals where power spectra are strictly in $(-a, a)$, any sampling plan regular enough can be associated with errorless formulas provided that the sampling rate is larger than $2 a / \pi$ [4-6]. They are close to Lagrange interpolation formulas, replacing far samples by well-chosen sequences. However this kind of scheme cannot be used when power spectra are not in baseband.

This paper addresses the problem of errorless sampling of stationary processes $\mathbf{Z}=\{Z(t), t \in \mathbb{R}\}$ with spectral support inside two symmetric sets of the real line. For instance and without loss of generality, let this support be (1), where $l \in \mathbb{N}^{*}$ and $\varepsilon>0$. This means that $[7,8]$

$$
\mathrm{E}\left[Z(t) Z^{*}(t-\tau)\right]=\int_{\Delta_{\varepsilon}} \mathrm{e}^{\mathrm{i} \omega \tau} S_{Z}(\omega) \mathrm{d} \omega
$$

where $\mathrm{E}[.$.$] stands for the mathematical expectation (or$ ensemble mean) and the superscript ${ }^{*}$ for the complex conjugate. $s_{Z}(\omega)$ is the spectral density of the process Z. We assume that $s_{Z}(\omega)=0$ in some neighboorhood of the bounds of $\Delta_{0}$ to be sure that $\mathbf{Z}$ is oversampled with respect to the Landau's theorem in all studied cases.

Knowing that $\varepsilon$ is arbitrary the minimal sampling rate for errorless reconstruction is 2 because $\left|\Delta_{0}\right|=4 \pi$ [1]. Samplings like PNSn (periodic nonuniform sampling of order n) may be solutions of the problem [9], [10]. For example we have the formula $[2,3]$

$$
\begin{gathered}
Z(t)=\frac{U_{a}(t) \sin 2 \pi l(t-b)+U_{b}(t) \sin 2 \pi l(a-t)}{\sin 2 \pi l(a-b)} \\
U_{x}(t)=\sum_{n \in \mathbb{Z}} \frac{\sin \pi(t-x-n)}{\pi(t-x-n)} Z(n+x)
\end{gathered}
$$

provided that $2 l(a-b) \notin \mathbb{Z}$. We are in the case of a PNS2 where the sampling sequences are $\mathbb{Z}+a$ and $\mathbb{Z}+b$.

$$
\Delta_{\varepsilon}=((-2 l-1) \pi+\varepsilon,(-2 l+1) \pi-\varepsilon) \cup((2 l-1) \pi+\varepsilon,(2 l+1) \pi-\varepsilon)
$$


In this paper we highlight a class of sampling sequences which answer the problem, and we give the associated reconstruction formulas. They generalize the PNS2 sampling set properties (and more generally the PNSn sets properties).

\section{A Sampling Formula}

Let $\mathbf{Z}$

$$
\mathbf{t}^{j}=\left\{t_{n}^{j}, n \in \mathbb{Z}\right\}, j=1,2
$$

be two sampling sequences with distinct elements $\left(t_{n}^{1} \neq t_{m}^{2}\right.$ whatever $\left.m, n\right)$ such that:

1) It exists two sampling formulas for the process in baseband of width $2 \pi$ defined by two kernels $g_{1}(t, x)$ and $g_{2}(t, x)$ :

$$
\left\{\begin{array}{l}
Z(t)=\sum_{n \in \mathbb{Z}} g_{j}\left(t, t_{n}^{j}\right) Z\left(t_{n}^{j}\right), j=1,2 \\
s_{Z}(\omega)=0 \text { for } \omega \notin(-\pi+\varepsilon, \pi-\varepsilon)
\end{array}\right.
$$

for some $\varepsilon>0$.

2) It exists two real numbers $\alpha_{1}, \alpha_{2}$ such that for all $n \in \mathbb{Z}$

$$
2 l t_{n}^{j} \in \mathbb{Z}+\alpha_{j}, \alpha_{2}-\alpha_{1} \notin \mathbb{Z} .
$$

At these conditions, and when $\left(\Delta_{\varepsilon}\right.$ is defined by (1))

$$
s_{Z}(\omega)=0, \omega \notin \Delta_{\varepsilon}
$$

we have the sampling formula (6).

The proof is in Appendix 1.

\section{Examples}

\subsection{Example 1}

The well-known formula (3) for the PNS2 verifies (6) with $(\operatorname{sinc} x=\sin x / x)$

$$
\left\{\begin{array}{c}
\alpha_{1}=2 l a, \alpha_{2}=2 l b, k_{n}^{1}=k_{n}^{2}=2 n l \\
g_{1}\left(t, t_{n}^{1}\right)=\sin \mathrm{c} \pi(t-a-n) \\
g_{2}\left(t, t_{n}^{1}\right)=\sin \mathrm{c} \pi(t-b-n) .
\end{array}\right.
$$

\subsection{Example 2}

Let consider the following sampling scheme

$$
t_{n}^{1}=n, t_{2 n}^{2}=2 n+a, t_{2 n+1}^{2}=2 n+a+\frac{1}{2}, 0<a<\frac{1}{2}
$$

(6) is available with [11,12],

$$
\left\{\begin{array}{c}
\alpha_{1}=0, k_{n}^{1}=2 n l, \alpha_{2}=2 l a, k_{2 n}^{2}=4 n l, k_{2 n+1}^{2}=(4 n+1) l \\
g_{1}\left(t, t_{n}^{1}\right)=\sin \mathrm{c} \pi(t-n) \\
g_{2}\left(t, t_{2 n}^{2}\right)=(-1)^{n} \sqrt{2} \sin \frac{\pi}{2}\left(t-a+\frac{1}{2}\right) \operatorname{sinc} \frac{\pi}{2}(t-a-2 n) \\
g_{2}\left(t, t_{2 n+1}^{2}\right)=(-1)^{n} \sqrt{2} \sin \frac{\pi}{2}(t-a) \operatorname{sinc} \frac{\pi}{2}\left(t-a-2 n-\frac{1}{2}\right)
\end{array}\right.
$$

Actually we are in the PNS4 frame with sequences based on $2 n, 2 n+a, 2 n+a+\frac{1}{2}, 2 n+1, n \in \mathbb{Z}$.

\subsection{Example 3}

We assume that

$$
t_{n}^{1}=n, t_{n}^{2}=n+a+b_{n}, b_{n} \in\left\{0, \pm \frac{1}{2 l}, \pm \frac{2}{2 l}, \ldots, \pm \frac{2 l-1}{2 l}\right\}
$$

with $2 \pi l a \notin \mathbb{Z}$ and the $b_{n}$ constant for $|n| \geq N>1$ (for instance equal to 0 ). We are no longer in the PNS frame (except when all $b_{n}$ are equal). We can take

$$
\left\{\begin{array}{c}
\alpha_{1}=0, k_{n}^{1}=2 n l, \alpha_{2}=2 l a, k_{n}^{2}=2 n l+2 b_{n} l \\
g_{1}\left(t, t_{n}^{1}\right)=\sin \mathrm{c} \pi(t-n)
\end{array}\right.
$$

where $g_{2}\left(t, t_{n}^{2}\right)$ is given by (7) (see the Appendix 2) with

$$
\frac{1}{F(z)}=\prod_{b_{n} \neq 0} \frac{\left(1-\frac{z}{n+a}\right)}{\left(1-\frac{z}{n+a+b_{n}}\right)} .
$$

If $l$ is large and then if the increments $1 / l$ are small,

$$
\begin{aligned}
& Z(t)=\frac{1}{\sin \pi\left(\alpha_{1}-\alpha_{2}\right)} \sum_{n \in \mathbb{Z}}(-1)^{k_{n}^{1}+k_{n}^{2}}\left[g_{1}\left(t, t_{n}^{1}\right) Z\left(t_{n}^{1}\right) \sin 2 \pi l\left(t-t_{n}^{2}\right)\right. \\
& \left.-g_{2}\left(t, t_{n}^{2}\right) Z\left(t_{n}^{2}\right) \sin 2 \pi l\left(t-t_{n}^{1}\right)\right] \\
& \alpha_{j}=2 l t_{n}^{j}-k_{n}^{j}, k_{n}^{j} \in \mathbb{Z} \text { and } \alpha_{2}-\alpha_{1} \notin \mathbb{Z} \\
& g_{2}\left(t, t_{n}^{2}\right)=F(t) \sin \pi(t-a)\left\{\begin{array}{c}
\frac{(-1)^{n}}{\left(t-a-n-b_{n}\right) F^{\prime}\left(n+a+b_{n}\right) \sin \pi b_{n}}, b_{n} \neq 0 \\
\frac{(-1)^{n}}{\pi(t-a-n) F(n+a)}, b_{n}=0 .
\end{array}\right.
\end{aligned}
$$


we have a model for (observed) jitter quantified at the value $1 / l$. Of course, we can complicate the sampling plan by introducing sampling gaps in the $t_{n}^{1}$.

\subsection{Example 4}

Examples above deal with two samplings $\mathbf{t}^{1}$ and $\mathbf{t}^{2}$ with equal mean rate 1 . Following the value of $l$ (the place of subbands) we can imagine samplings with mean rates which are different and not multiple (but rational between them). For instance consider the following case

$$
t_{n}^{1}=n, t_{n}^{2}=\frac{2 n}{3}+a, l \in 3 \mathbb{Z}, 2 l a \notin \mathbb{Z} .
$$

This corresponds to

$$
\left\{\begin{array}{c}
\alpha_{1}=0, k_{n}^{1}=2 n l, \alpha_{2}=2 l a, k_{n}^{2}=\frac{4}{3} n l \\
g_{1}\left(t, t_{n}^{1}\right)=\sin \mathrm{c} \pi(t-n) \\
g_{2}\left(t, t_{n}^{2}\right)=\sin \mathrm{c} \frac{3 \pi}{2}\left(t-a-\frac{2 n}{3}\right)
\end{array}\right.
$$

$g_{2}\left(t, t_{n}^{2}\right)$ is the usual sampling formula matched to the sampling rate $3 / 2$ delayed by $a$, true for power spectra in $(-3 \pi / 2,3 \pi / 2)$. The larger $l$ the better the choice for available samplings. Unlike the preceeding examples, we are in a situation of a true oversampling ( $\varepsilon$ is arbitrarily small). However, if $l$ is not too small, the mean rate sampling is more favourable than the Nyquist one.

\subsection{Example 5}

One or both sequences can be mixed. For instance

$$
t_{n}^{1}=\left\{\begin{array}{l}
n \text { for even } n \\
\frac{3 n}{4} \text { for odd } n
\end{array}, t_{n}^{2}=n+a, l \in 2 \mathbb{Z}, 2 l a \notin \mathbb{Z} .\right.
$$

The formula (6) can be used when $l \in 2 \mathbb{Z}, 2 l a \notin \mathbb{Z}$, with

$$
\left\{\begin{aligned}
& \alpha_{1}=0, k_{2 n}^{1}=4 n l, k_{2 n+1}^{1}=\frac{3}{2} l(2 n+1), \alpha_{2}=2 l a, k_{n}^{2}=2 n l \\
& g_{1}\left(t, t_{n}^{1}\right)=\sin \frac{\pi t}{2} \cos \frac{2 \pi t}{3}\left\{\begin{array}{l}
\frac{2}{\pi(t-n) \cos (\pi n / 6)} \text { for even } n \\
\frac{-3}{\pi(t-n) \cos (\pi n / 8)} \text { for odd } n
\end{array}\right. \\
& g_{2}\left(t, t_{n}^{2}\right)=\sin \operatorname{c} \pi(t-n-a)
\end{aligned}\right.
$$

\section{Conclusions}

Most of the time, processes used in communications occupy symmetrical power spectral bands in the form $\Delta=(-b,-a) \cup(a, b), a>0$. Very often, the relative bandwidth $2(b-a) / b$ is small. However, most of the sam- pling formulae are matched to baseband processes where $\Delta=(-a, a)$. In this case the choice of errorless samplings is large, whatever the sampling, uniform or irregular $[5,6$, 13]. The sampling mean rate for errorless reconstruction is $a / \pi$ in the latter case (the Nyquist rate) and it is $(b-a) / \pi$ in the former case (the Landau rate) [1]. In communications the Landau rate is small in front of the Nyquist rate. The research for errorless samplings with Landau rate is important for reducing calculus cost. The choice of errorless samplings is limited to the PNS $[9,10]$ and has to be increased. It is the aim of this short paper. A new sampling formula is proved and examples are given. They are based on formulas true in baseband and generally well-known [4,14,15]. Example 3 deals with irregular samplings at the Landau rate and can be used in the presence of jitter. In example 4, we have two samplings with different periods which generalizes the PNS2. The method which is used can be generalized to other power spectra including more than two pieces $[16,17]$. It is also possible to use a mixing of several periodic samplings for the sequences $\mathbf{t}^{1}$ and/or $\mathbf{t}^{2}[11,12]$.

\section{REFERENCES}

[1] H. J. Landau, "Sampling, Data Transmission, and the Nyquist Rate," Proceedings of the IEEE, Vol. 55, No. 10, 1967, pp. 1701-1706. doi:10.1109/PROC.1967.5962

[2] B. Lacaze, "About Bi-Periodic Samplings," Sampling Theory in Signal and Image Processing, Vol. 8, No. 3, 2009, pp. 287-306.

[3] B. Lacaze, "Equivalent Circuits for the PNS2 Sampling Scheme," IEEE Circuits and Systems, Vol. 57, No. 11, 2010, pp. 2904-2914. doi:10.1109/TCSI.2010.2050228

[4] A. J. Jerri, "The Shannon Sampling Theorem. Its Various Extensions and Applications. A Tutorial Review," Proceedings of the IEEE, Vol. 65, No. 11, 1977, pp. 15651596. doi:10.1109/PROC.1977.10771

[5] B. Lacaze, "Reconstruction Formula for Irregular Sampling," Sampling Theory in Signal and Image Processing, Vol. 4, No. 1, 2005, pp. 33-43.

[6] B. Lacaze, "The Ghost Sampling Sequence Method," Sampling Theory in Signal and Image Processing, Vol. 8, No. 1, 2009, pp. 13-21.

[7] H. Cramér and M. R. Leadbetter, "Stationary and Related Stochastic Processes," Wiley, New York, 1966.

[8] A. Papoulis, "Signal Analysis," McGraw Hill, New York, 1977.

[9] J. R. Higgins, "A Sampling Theorem for Irregular Sample Points," IEEE Transactions on Information Theory, Vol. 22, No. 5, 1976, pp. 621-622. doi:10.1109/TIT.1976.1055596

[10] J. R. Higgins, "Some Gap Sampling Series for Multiband Signals," Signal Processing, Vol. 12, No. 3, 1987, pp. 313319. doi:10.1016/0165-1684(87)90100-9 
[11] J. L. Yen, "On Nonuniform Sampling of BandwidthLimited Signals," IRE Transactions on Circuit Theory, Vol. 3, No. 4, 1956, pp. 251-257.

[12] B. Lacaze, "About a Multiperiodic Sampling Scheme," IEEE Signal Processing Letters, Vol. 6, No. 12, 1999, pp. 307-308. doi:10.1109/97.803430

[13] B. Lacaze, "A Theoretical Exposition of Stationary Processes Sampling," Sampling Theory in Signal and Image Processing, Vol. 4, No. 3, 2005, pp. 201-230.

[14] K. Seip, "An Irregular Sampling Theorem for Functions Bandlimited in a Generalized Sense," SIAM Journal on Applied Mathematics, Vol. 47, No. 5, 1987, pp. 11121116. doi: $10.1137 / 0147073$

\section{Appendix}

\section{Appendix 1}

$$
\begin{aligned}
& \text { If } h_{j}^{m}(t, \omega)=\sum_{n=-m}^{m} g_{j}\left(t, t_{n}^{j}\right) \mathrm{e}^{i \omega t_{n}^{j}} \quad \text { (4) is equivalent to } \\
& \qquad \lim _{m \rightarrow \infty} \int_{-\pi+\varepsilon}^{\pi-\varepsilon}\left|\mathrm{e}^{i \omega t}-h_{j}^{m}(t, \omega)\right|^{2} S_{Z}(\omega) \mathrm{d} \omega=0 .
\end{aligned}
$$

Then we also have

$$
Z(t)=\mathrm{e}^{i a t} \sum_{n \in \mathbb{Z}} \mathrm{e}^{-i a t_{n}^{j}} g_{j}\left(t, t_{n}^{j}\right) Z\left(t_{n}^{j}\right)
$$

when

$$
s_{Z}(\omega)=0 \text { for } \omega \notin(-\pi+a+\varepsilon, \pi+a-\varepsilon) .
$$

Now we assume that $s_{Z}(\omega)=0$ for $\omega \notin \Delta_{\varepsilon}$. We write:

$$
\left\{\begin{array}{c}
Z(t)=Z_{+}(t)+Z_{-}(t) \\
s_{Z_{+}}(\omega)=0, \omega \notin((2 l-1) \pi,(2 l+1) \pi) \\
s_{Z_{-}}(\omega)=0, \omega \notin((-2 l-1) \pi,(-2 l+1) \pi) .
\end{array}\right.
$$

We define (11)

From (9), (10) we have

$$
A\left(t, \alpha_{j}\right)=\mathrm{e}^{-2 i \pi l t} Z_{+}(t)+\mathrm{e}^{-2 i \pi \alpha_{j}+2 i \pi l t} Z_{-}(t) .
$$

When

$$
\alpha_{j}=2 l t_{n}^{j}-k_{n}^{j}, k_{n}^{j} \in \mathbb{Z} \text {, whatever } n \in \mathbb{Z}
$$

we have

$$
A\left(t, \alpha_{j}\right)=\mathrm{e}^{-i \pi \alpha_{j}} \sum_{n \in \mathbb{Z}}(-1)^{k_{n}^{j}} g_{j}\left(t, t_{n}^{j}\right) Z\left(t_{n}^{j}\right) .
$$

[15] A. I. Zayed and P. L. Butzer, "Lagrange Interpolation and Sampling Theorems," In: F. Marvasti, Ed., Nonuniform Sampling: Theory and Practice, Kluwer Academic, 2001, pp. 123-168. doi:10.1007/978-1-4615-1229-5 3

[16] Y.-P. Lin and P. P. Vaidyanathan, "Periodically Nonuniform Sampling of Bandpass Signals," IEEE Transactions on Circuits and Systems II, Vol. 45, No. 3, 1998, pp. 340351.

[17] R. Venkataramani, "Perfect Reconstruction Formulas and Bounds on Aliasing Error in Sub-Nyquist Nonuniform Sampling of Multibands Signals," IEEE Transactions on Information Theory, Vol. 46, No. 6, 2000, pp. 2173-2183.

The linear system $(12,13)$ is solved in (14) which leads to (6). We understand the key of this formula. The problem is to write (1) so that $Z_{+}\left(t_{n}^{j}\right)$ and $Z_{-}\left(t_{n}^{j}\right)$ disappear (they are not observed) and the $Z\left(t_{n}^{j}\right)$ appear (they are observed).

\section{Appendix 2}

With $F(z)$ defined by (8) we consider $I_{n}$ the complex integral

$$
I_{n}=\int_{C_{n}} \frac{\mathrm{e}^{i \omega z}}{(z-t) F(z) \sin \pi(z-a)} \mathrm{d} z
$$

where $C_{n}$ is the square crossing the axes $\mathrm{Ox}, \mathrm{Oy}$ at the points $|x|=n+a+1 / 2,|y|=n+1 / 2$. Because

$$
\lim _{|z| \rightarrow \infty} F(z)=\gamma<\infty
$$

we also have for $\omega \in(-\pi+\varepsilon, \pi-\varepsilon) \lim _{n \rightarrow \infty} I_{n}=0$.

We apply the residue's theorem with order 1 singularities at the points $n+a+b_{n}$ with $b_{n}=0$ for $|n|>N$. Consequently

$$
\begin{aligned}
& \frac{\mathrm{e}^{i \omega t}}{F(t) \sin \pi t}=\sum_{b_{n}=0} \frac{(-1)^{n} \mathrm{e}^{i \omega(n+a)}}{\pi(t-a-n) F(n+a)} \\
& +\sum_{b_{n} \neq 0} \frac{(-1)^{n} \mathrm{e}^{i \omega\left(n+a+b_{n}\right)}}{\left(t-a-n-b_{n}\right) F^{\prime}\left(n+a+b_{n}\right) \sin \left(b_{n}\right)} .
\end{aligned}
$$

We obtain (7) from (15) using the fundamental isometry which allows to change $\mathrm{e}^{i \omega x}$ by $Z(x)$ in (15) [13].

$$
\begin{gathered}
A\left(t, \alpha_{j}\right)=\sum_{n \in \mathbb{Z}} g_{j}\left(t, t_{n}^{j}\right)\left[\mathrm{e}^{-2 i \pi l t_{n}^{j}} Z_{+}\left(t_{n}^{j}\right)+\mathrm{e}^{-2 i \pi \alpha_{j}+2 i \pi l t_{n}^{j}} Z_{-}\left(t_{n}^{j}\right)\right] . \\
\left\{\begin{array}{c}
Z(t)=\frac{1}{\sin \left(\alpha_{2}-\alpha_{1}\right)}\left[A\left(t, \alpha_{1}\right) \mathrm{e}^{i \pi \alpha_{1}} \sin \pi\left(\alpha_{2}-2 l t\right)-A\left(t, \alpha_{2}\right) \mathrm{e}^{i \pi \alpha_{2}} \sin \pi\left(\alpha_{1}-2 l t\right)\right] \\
\alpha_{j}=2 l t_{n}^{j}-k_{n}^{j}, k_{n}^{j} \in \mathbb{Z}
\end{array}\right.
\end{gathered}
$$

